\title{
Modulator and filter circuits for EEG biofeedback
}

\author{
R. J. HELMER \\ Trinity University, San Antonio, Texas 78284
}

\begin{abstract}
Inexpensive designs for two components of an EEG biofeedback system are described. A two-channel modulator accepts a pair of input signals for modulation of two audio carrier frequencies a musical fifth apart. Carrier suppression is obtained with a simple balanced modulator circuit for each channel. The filter circuit is an integrated circuit adaptation of a successful vacuum tube design reported by other investigators a number of years ago. Various configurations of this equipment for two-channel EEG biofeedback are described.
\end{abstract}

A two-channel signal processing system for EEG biofeedback makes possible several experimental configurations suitable for student projects, demonstrations, or research. The equipment described here was designed to provide flexibility of operation and allow one or two channels of feedback. With two channels, the unit can supply separate signals, if required, for the operation of amplifiers driving stereo headphones.

\section{BALANCED MODULATORS}

The modulation process for each channel is of the suppressed carrier, double sideband type. This system is well known in communication engineering but is not generally familiar to students of the behavioral sciences. The suitability of this type of modulation for EEG biofeedback can be illustrated by a simple example. Consider a low frequency signal of physiological origin. It is in the subaudible frequency range and has a slowly varying peak amplitude, that may take on values from zero up to some maximum value. This signal can be used to modulate a carrier wave chosen for agreeable listening qualities-say a $500-\mathrm{Hz}$ sine wave. For example, assume the physiological signal to be a $5-\mathrm{Hz}$ sine wave for the time interval under consideration. A straightforward amplitude modulation process would use the low frequency signal to control the amplitude of the higher frequency sine wave, causing it to vary above and below some original (unmodulated) value. The result is a useful transformation of the subaudible signal by which some of its characteristics can be made audible. The disadvantage of this basic type of amplitude modulation is the presence of a constant component (the carrier), whether the modulating signal is high or low in amplitude, or absent. It is more suitable to have a system with no output when there is no modulating signal, and a system that produces an output proportional to the input. In mathematical terms, this suggests subtracting from the amplitude modulated signal the amount of carrier energy which corresponds to zero modulating signal. This interpretation clarifies the reference to a suppressed carrier. Double sidebands are fundamental to the basic amplitude modulation process; in the example chosen, they are of equal amplitude, with frequencies of 495 and $505 \mathrm{~Hz}$. The corresponding sound can be described either in terms of a beat note or a tremolo effect, but the utility, if any, of such a process can be evaluated only by listening tests. These are made with various types of EEG signals, both filtered and unfiltered, as modulating signals.

The unit described here is a two-channel device with a pair of identical modulators. The two modulating signal inputs are, in general, different waveforms derived from one or more EEG signals. The two channels differ only in the values of the carrier frequencies supplied to their respective modulators, and their outputs are aurally distinguishable on that basis. Values of $500 \mathrm{~Hz}$ and $750 \mathrm{~Hz}$ were chosen for the carrier frequencies. These are a musical fifth apart and the final outputs of the modulators, as derived from these frequencies, have approximately the same relationship. This arrangement gives a pair of easily distinguished tones without dissonance. With two EEG signals most observers report that they can listen selectively to one pitch or the other with little or no practice, with monophonic presentation through a single loudspeaker.

Figure I shows the balanced modulator circuit, including a generator for the two carrier frequencies. A multivibrator based on two cross-coupled $74121 \mathrm{~s}$ generates a $3-\mathrm{KHz}$ square wave to be divided in frequency to $500 \mathrm{~Hz}$ and $750 \mathrm{~Hz}$ by two parallel divider chains. Only the $500-\mathrm{Hz}$ modulator, driven by the upper divider, is shown. The $750-\mathrm{H} z$ circuit is identical except for the values of its three equal capacitors in the low-pass RC network in the output circuit. The 3-KHz multivibrator employs no critical components. The two sections need not be matched; the divided-by-two operation in each chain assures that a 50\% duty-cycle waveform is supplied to each modulator. A "starter" circuit connected to the multivibrator assures that it will not turn on and remain in a stable, inoperative state when power is applied. This difficulty may be encountered with power supplies that have a slow voltage rise when activated. In an inoperative state both Q outputs would be at a logical "l" level. If this happens, 


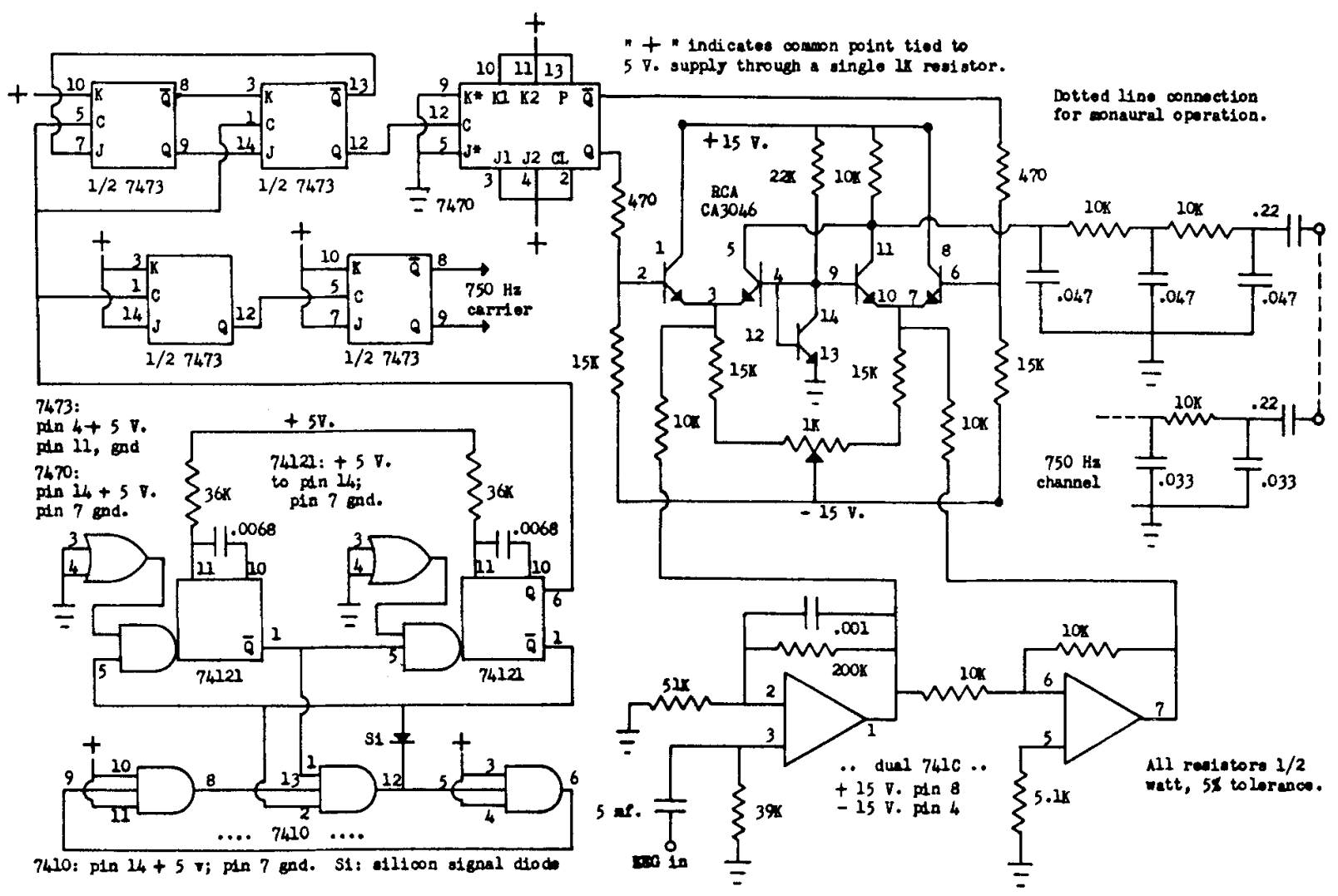

Figure 1. The dual modulator. For monophonic use the outputs are combined by making the dotted line connection. Otherwise the two outputs are fed to the inputs of a stereo amplifier.

it allows a ring oscillator ${ }^{1}$, formed by the three sections of the 7410 , to produce a $20-\mathrm{MHz}$ (typical) output that can momentarily reduce the voltage at the anode of the silicon diode, thus forcing the right-hand half of the multivibrator to change state. Normal operation then begins, and the ring oscillator is turned off because one or the other of the inputs, 1,2 of the 7410 , will be at logical " 0 " level at a given instant. The low cost of the components used to construct the dual carrier generator makes the use of laboratory instruments for this purpose an unnecessary complication. A more basic consideration is the requirement for very closely matched push-pull waveforms with fast TTL switching times. This makes a very simple balancing system possible.

Suppressed carrier modulation is obtained with transistors in the integrated circuit N-P-N array RCA CA3046. Transistors 5, 4, 3 and 11, 9, 10 (14-pin DIP numbers) are switched on alternately by the carrier signal. With no modulation, the on currents are balanced using the $1 \mathrm{~K}$ trimmer potentiometer. A null is then obtained at the common collector point 5, 11. The adjustment can be made by listening to the output using an amplifier and loudspeaker.

The EEG input signal should be of 1-2 V peak amplitude (nominal $1.4 \mathrm{~V}$ ). This is the modulating signal that is first amplified and phase-inverted by the operational amplifiers. The two resulting signals are coupled to the emitters of the switched transistors. A nonzero modulating signal produces a current unbalance in the switched transistors. This appears as a modulation envelope of the carrier with phase reversal of the modulating signal causing a phase reversal of the carrier. This waveform appears at the common collector point 5 , 11. An examination of the carrier switching circuit shows that for a half-cycle either transistor $1,2,3$ or 8 , 6,7 will conduct and as its emitter voltage rises it will turn off the switched transistor with which it shares a common emitter connection. On the following half-cycle the same transistor $(1,2,3$, or $8,6,7)$ will be turned off by the carrier. It then has no effect on the switched transistor. The latter will be in normal conduction with a collector current determined by circuit values and the instantaneous modulating voltage. The total collector current of the two switched transistors is the desired suppressed carrier, amplitude modulated signal except for the presence of harmonics of the carrier frequency. Harmonic attenuation is obtained by a simple 3-section low pass network with capacitor values appropriate to the carrier frequency of each modulator. Since the unfiltered signal (a square wave) has no even-order harmonics and since the carrier frequencies are in $3: 2$ 


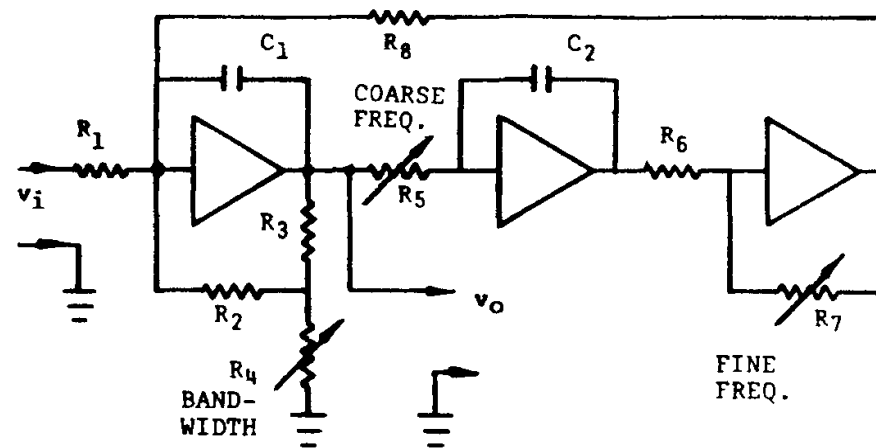

$\underset{\text { FREQUENCY }}{\text { CENTER }} \quad f_{0}=\frac{1}{2 \pi} \sqrt{\frac{R_{7}}{R_{5} R_{6} R_{8} C_{1} C_{2}}} \mathrm{HZ}$

$\begin{aligned} & \text { BANDWIDTH (BETWEEN } \\ & \text { 3-DB POINTS), HZ. }\end{aligned} \quad B=\frac{R_{4}}{2 \pi R_{2} C_{1}\left(R_{3}+R_{4}\right)}$

FOR $R_{3} \gg R_{4}, B=\frac{R_{4}}{2 \pi R_{2} C_{7} R_{3}}$

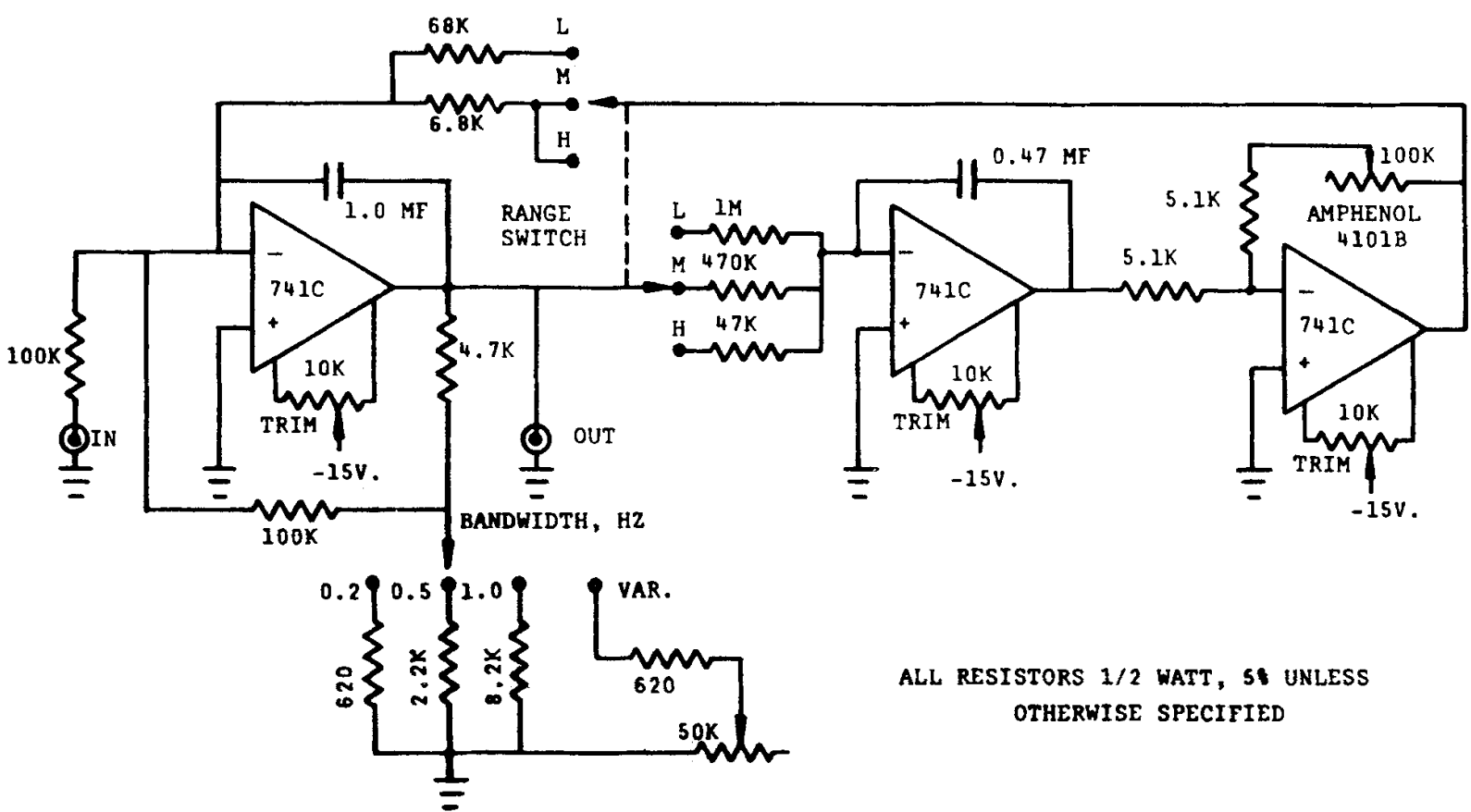

Figure 2. Filter design parameters are shown, with an example of inexpensive IC design.

ratio, there is no harmonic frequency common to the two channels. This assures that there is no ambiguity in the combined signal from the two modulators as far as separation of channels is concerned.

\section{OPTIONS}

The modulator used alone, without filters or nonlinear circuits, may be suitable for some projects or demonstrations involving one or two EEG channels. For example, the characteristic musically pitched rattle produced by the loudspeaker as the result of an alpha train in the signal is easily recognized, even though the bandwidth of the EEG input is limited only by cutoff values such as $1 \mathrm{~Hz}$ and $30 \mathrm{~Hz}$ for the low and high frequency limits.

Threshold or limiting circuits have not been used with this equipment. Presumably, most applications will be based on recognition of relatively subtle differences in sound by the subject, which is somewhat at variance with the "on-off" operation of threshold or triggering circuits. If the user wishes to incorporate threshold capability, the circuits ${ }^{2}$ should be interposed between the filters, if used, and the modulators.

Dual modulator operation does not necessarily require two EEG signals for practical application. A pair of tuned filters can be used to separate different frequency components from a single preamplifier signal and provide distinguishable tones in accordance with the frequency content of the single channel. An interesting example of this involves use of a filter to drive one input of the modulator with theta signals and a second filter to drive the other input of the modulator with alpha signals, both from the same EEG electrodes.

To realize the full system flexibility, two filters and two EEG preamplifier systems are required. The latter must supply appropriately band limited (e.g., 1 to $30 \mathrm{~Hz}$ ) signals at $1 \mathrm{~V}$ amplitude.

Extension of the system to four channels by means of diphonic presentation at two musical pitches is clearly 
possible, but no evaluation of this scheme has been made with the equipment described here.

\section{FILTERS}

An integrated circuit adaptation of a well-known vacuum tube design (Lowenberg \& McCullough, 1963) has been found to be quite satisfactory for present purposes. Filters of this type have an amplitude vs. frequency response characteristic of a single tuned circuit with constant (selectable) bandwidth. In Figure 2, the basic design parameters, summarized from the report describing the original circuit (op. cit) are shown along with a circuit based on contemporary IC operational amplifiers. The IC design retains the specifications of the vacuum-tube prototype with regard to frequency range ( $1 \mathrm{~Hz}$ to $60 \mathrm{~Hz}$ in three ranges) and fixed bandwidth values selectable by switching. Intermediate values are obtained by the variable bandwidth control. The filter has a nominal gain of one, obtained at wider bandwidths, increasing with the sharpness of tuning. An input of $1 \mathrm{~V}$ to the filter provides an output signal to drive the modulator of Figure 1. At $1-\mathrm{Hz}$ bandwidth (the most useful mode of operation) attenuation is not necessary; at .5 or $.2 \mathrm{~Hz}$, attenuation before or after the filter should be provided to give a modulator input of approximately $1 \mathrm{~V}$.

Ultimate performance of the circuit requires the use of precision metal film resistors and polystyrene capacitors. For EEG work, composition carbon resistors and mylar capacitors appear to be entirely satisfactory. The minature 10-turn potentiometer with a turns-counting dial is almost indispensable for quick reproducibility of settings. This is of importance, for example, in a situation where a subject is led to produce progressively lower alpha frequencies by means of small changes in filter tuning.

\section{CONSTRUCTION NOTES: COSTS}

The dual modulator, less power supply, can be constructed for approximately $\$ 60$ in parts cost, including IC sockets, the use of which is recommended. Radio Shack ICs were used in all positions except for the RCA CA3046s. The Radio Shack types have the advantage of quick availability with the drawback of some shrinkage due to an occasional defective unit. These can usually be identified immediately and discarded when the circuit is tested. Texas Instruments ICs may be substituted for the Radio Shack digital items.

Parts cost for the tunable filter, less power supply, is estimated at $\$ 25$; about half of the cost is for the 10-turn potentiometer and miniature turns-counting dial. Given an overriding requirement for economy, these items could be replaced by a resistor switching arrangement, at some loss of convenience. Operational amplifiers, capacitors, switches and the $50 \mathrm{~K}$ control are Radio Shack items.

\section{REFERENCE}

Lowenberg, Edwin C., \& McCullough, Charles E., An improved Hi-Q bandpass filter for electroencephalography Electroencephalography and Clinical Neurophysiology, 1963, 15, 706-708.

\section{NOTES}

1. The usefulness of the single IC gated ring oscillator was noted in a flurry of brief construction notes on the advent of the IC triple-nand gate. See, for example, a contribution by Wise and IC triple-nand gate. See, for example, a contribution by Wise and Design, Vol. 2, p. 118; Hayden Book Company, New York.

2 Rough notes with suggestions for simple circuits can be furnished by the author to users who are not familiar with the usual techniques.

(Received for publication October 31,1974 , revision received December $7,1974$. ) 